\title{
Partially hydrogenated and fluorinated graphene: Structure, roughness, and negative thermal expansion
}

\author{
M. Neek-Amal ${ }^{1,2}$ and F. M. Peeters ${ }^{2}$ \\ ${ }^{1}$ Department of Physics, Shahid Rajaee Teacher Training University, Lavizan, Tehran 16785-136, Iran \\ ${ }^{2}$ Department of Physics, University of Antwerpen, Groenenborgerlaan 171, B-2020 Antwerpen, Belgium
}

(Received 5 June 2015; revised manuscript received 7 October 2015; published 23 October 2015)

\begin{abstract}
The structural properties of partially hydrogenated and fluorinated graphene with different percentages of $\mathrm{H} / \mathrm{F}$ atoms are investigated using molecular dynamics simulations based on reactive force field (ReaxFF) potentials. We found that the roughness of graphene varies with the percentage $(p)$ of $\mathrm{H}$ or $\mathrm{F}$ and in both cases is maximal around $p=50 \%$. Similar results were obtained for partially oxidized graphene. The two-dimensional area size of partially fluorinated and hydrogenated graphene exhibits a local minimum around $p=35 \%$ coverage. The lattice thermal contraction in partially functionalized graphene is found to be one order of magnitude larger than that of fully covered graphene. We also show that the armchair structure for graphene oxide (similar to the structure of fully hydrogenated and fluorinated graphene) is unstable. Our results show that the structure of partially functionalized graphene changes nontrivially with the $\mathrm{C}: \mathrm{H}$ and $\mathrm{C}: \mathrm{F}$ ratio as well as with temperature.
\end{abstract}

DOI: $10.1103 /$ PhysRevB.92.155430

PACS number(s): 65.40.De

\section{INTRODUCTION}

Graphene is a crystalline allotrope of carbon arranged in a two-dimensional (2D) honeycomb lattice with $s p^{2}$ in-plane covalent bonds [1,2], which has gained considerable attention in recent decades due to its exceptional physical properties [3-8]. The thermal properties of graphene are of particular interest because it has the largest thermal conductivity among other materials, i.e., $1000 \mathrm{~W} \mathrm{~m}^{-1} \mathrm{~K}^{-1}$. Therefore, it is a desirable candidate in applications for thermal transport and heat management in nanoelectronic devices [9]. Experimental observations have found ripples in suspended sheets of graphene [10], and recently our atomistic simulations suggest that the strong bonds between the carbon atoms determine the features of these ripples [11].

On the other hand, hydrocarbons $(\mathrm{CH})_{n}$ are the simplest organic structures, made of merely carbon and hydrogen atoms [12], which have a very different thermal conductivity due to $s p^{3}$ bond formation in hydrogenated graphene. Experimentally, it has been shown that hydrogenated graphene can be obtained reversibly starting from a pure graphene layer [13], and since then it has become a material of high interest due to its potential applications in nanoelectronics [14]. We recently found that fully hydrogenated graphene is an unrippled system in contrast to graphene [15], with a lower melting temperature than graphene [16]. However, experimentally, information about the rippling of partially hydrogenated graphene and on its lattice expansion/contraction is lacking (more properties of hydrogenated graphene can be found in a recent review paper, i.e., Ref. [17]).

In addition to hydrogenated graphene, recently, a similar functionalization was realized by adding $\mathrm{F}$ atoms [18] to graphene. Since the $\mathrm{F}$ atoms have larger binding and desorption energies than $\mathrm{H}$ atoms [18], the C-F bonds are energetically more stable than the $\mathrm{C}-\mathrm{H}$ bonds [19-21]. Both theory and experiment report the stability of one-sided fluorinated graphene $[22,23]$ while one-sided fluorinated graphene nanoribbons are found to curl up into nanotubelike structures [24]. Furthermore, fluorination is easier to control via temperature and reactant gases, leading to better reproducibility with precise $\mathrm{C} / \mathrm{F}$ stoichiometries $[25,26]$.
In the presence of $\mathrm{F} / \mathrm{H}$ adatoms, $\mathrm{C}$ bonds in graphene transit from $s p^{2}$ to $s p^{3}$ hybridization, which turns the conjugated graphitic C-C bonds into single C-C bonds. This rehybridization turns the lattice structure into an angstrom scale out-of-plane buckled membrane known commonly as the chair configuration [19,21]. The buckling of fully covered graphene by $\mathrm{H}(\mathrm{F})$ has an out-of-plane deformation of about $0.044 \mathrm{~nm}(0.045 \mathrm{~nm})$.

In this paper, using state-of-the-art molecular dynamics (MD) simulations, we show that the roughness of graphene varies strongly with the $\mathrm{C}: \mathrm{H}$ and $\mathrm{C}: \mathrm{F}$ ratio. We found that a random distribution of $\mathrm{H} / \mathrm{F}$ atoms over graphene changes significantly the morphology of the system and can change the size of the samples. In fact, there are two different contributions to the rippling structure in partially functionalized graphene, i.e., thermal and structural. The thermal contribution of the ripples is one order of magnitude larger than that of the structural contribution. The energy of the carbons in hydrogenated (fluorinated) graphene decreases (increases up to $35 \%$ and then decreases) with the concentration $p$. We found that the area of partially hydrogenated/fluorinated graphene is minimum at around $35 \%$, and a negative thermal expansion is found for partially functionalized graphene.

The paper is organized as follows. In Sec. II we review the method used and the presented model. In Sec. III the roughnesses of partially hydrogenated and fluorinated graphene at zero and finite temperature are reported and compared with partially oxidized graphene. Section IV contains results for the binding energy. In Secs. V and VI the in-plane size deformations of partially functionalized graphene at zero and finite temperature are reported, respectively. The thermal contraction is investigated in Sec. VII. Our results are discussed in Sec. VIII.

\section{THE MODEL AND COMPUTATIONAL METHODS}

The partially hydrogenated/fluorinated/oxidized systems are generated by randomly removing $\mathrm{H} / \mathrm{F} / \mathrm{O}$ atoms from both sides of the fully covered systems. The initial configuration of perfect graphene, fully hydrogenated graphene (chairlike 

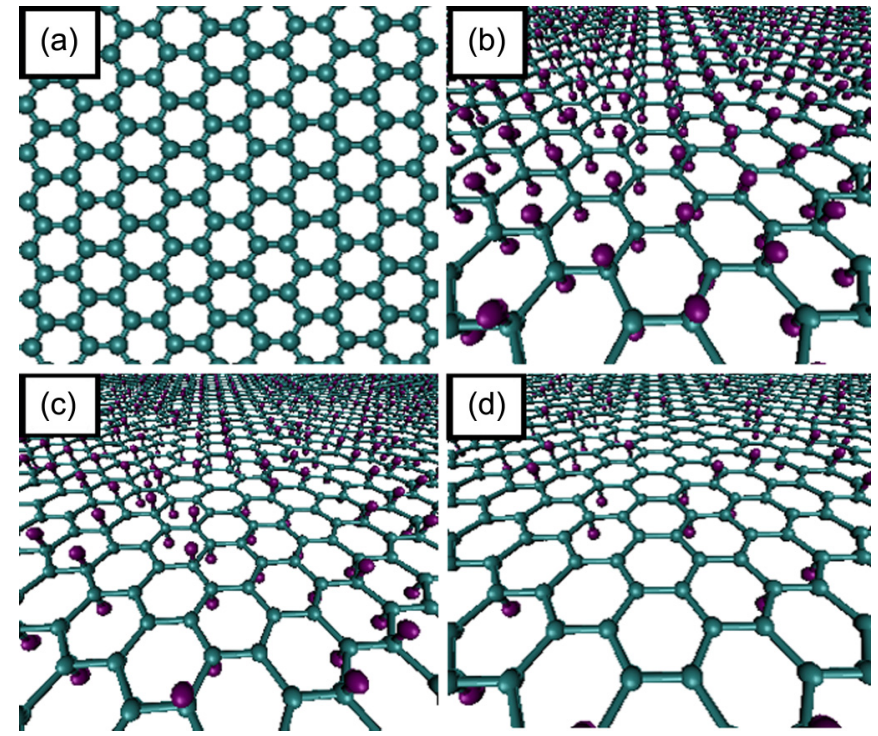

FIG. 1. (Color online) (a) Perfect graphene (top view), and hydrogenated graphene with (b) $100 \%$, (c) $50 \%$, and (d) $25 \%$ concentrations of hydrogen atoms (side view).

configuration), and graphene with $p=50 \%$ and $25 \%$ hydrogens are shown in Figs. 1(a)-1(d). Those figures are qualitatively the same if we replace $\mathrm{H}$ by $\mathrm{F}$ or $\mathrm{O}$.

In order to study the ripples caused by the $\mathrm{H}(\mathrm{F}, \mathrm{O})$ atoms over graphene, we performed several MD simulations for a system with $12000(11200,12000)$ carbon atoms with different percentages of randomly distributed $\mathrm{H}(\mathrm{F}, \mathrm{O})$ [the total number of atoms, e.g., in fully hydrogenated and oxidized (fluorinated) graphene is 24000 (22 400) atoms]. We employed periodic boundary conditions in our molecular dynamics (MD) simulations using reactive force fields (ReaxFF [27-29]). The large-scale atomic/molecular massively parallel simulator (LAMMPS) code was used [30,31]. The ReaxFF potential is a reactive force field that accounts for possible bond formation and bond dissociation of different bond orders. It has been shown that this potential describes correctly the thermal properties of fluorographene [32]. The new set of parameters appropriate for structures with C-F bonds can be found in our previous study [32]. The MD simulations for graphene-oxygen systems with ReaxFF gives energies, transition states, reaction pathways, and reactivity trends for hydrocarbon-oxygen that are in agreement with quantum mechanical calculations and experiments [33]. It was shown that ReaxFF gives reasonable results for hydrogen bond networks in graphene oxide composites [34] as well as its structural evolution during the reduction [35]. There are different studies about the bonding mechanism between graphene and oxygen, and most of them propose the formation of oxygen functional groups on graphene, such as epoxy and hydroxyl molecules. There is also evidence for the presence of ketones and phenols [35]. Despite these previous models, a systematic study of the structure of graphene oxide is still lacking. Here, we report on the instability of oxidized graphene with an armchair configuration. Such an armchair configuration is similar to the structure of graphane and fluorinated graphene. Nevertheless, we included the graphene with oxygen system for comparative purposes.
In this paper we find the minimum energy configuration of hydrogenated/fluorinated/oxidized graphene using periodic boundary conditions. Before minimizing the energy, we perform annealing molecular dynamics simulations using the $N P T$ ensemble. The annealing simulations starts at $T=10 \mathrm{~K}$ and ends at $T=0 \mathrm{~K}$ using a $2 \mathrm{~K} / \mathrm{ps}$ rate at zero pressure. This is done in order to remove boundary stress and to find the true simulation box size. After that, the total energy is minimized using the iterative conjugate gradient (CG) scheme. The CG method in LAMMPS uses the Polak-Ribiere algorithm, which at each iteration combines the force gradient with the previous iteration information to compute a new search direction that is perpendicular (conjugate) to the previous search direction. As a result, this gives us the proper configuration of the system for different concentrations of hydrogen/fluorine/oxygen atoms. We used a time step of 0.1 fs during annealing. A NoséHoover thermostat and barostat [36,37] were used to control the temperature and to keep the pressure constant. Note that the barostat used should only scale the $x-y$ coordinates of the atoms. For this reason we used a nonisotropic barostat in our simulations.

In order to calculate the thermal expansion, we heat the system starting at $0 \mathrm{~K}$ up to $600 \mathrm{~K}$ at a rate of $2 \mathrm{~K} / \mathrm{ps}$. We save the dimension of the system and calculate the size variation with respect to the size at $0 \mathrm{~K}$ and estimate the thermal expansion coefficient of the system.

\section{RIPPLES IN PARTIALLY HYDROGENATED/FLUORINATED GRAPHENE}

By relaxing the system we reach the system equilibrium state, after which we calculate the roughness using

$$
\sigma^{2}=\left\langle z^{2}\right\rangle-\langle z\rangle^{2},
$$

where $\langle z\rangle$ and $\left\langle z^{2}\right\rangle$ are the average values of the height and square height of the carbon atoms, respectively. We

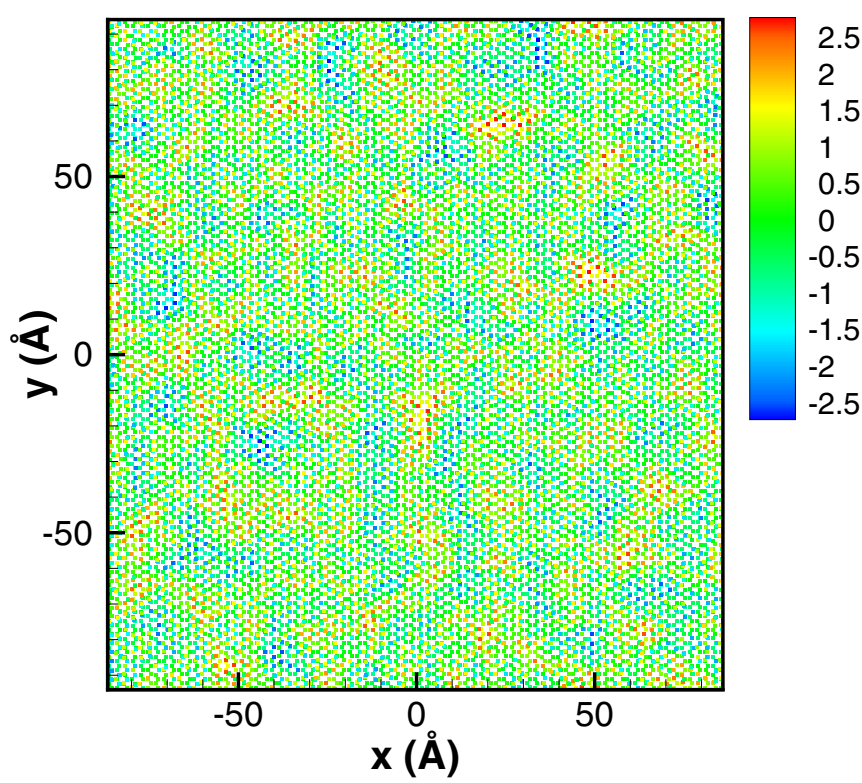

FIG. 2. (Color online) Plot of the out-of-plane position (in units of angstrom) of the $\mathrm{C}$ atoms with $50 \%$ randomly distributed hydrogens. 


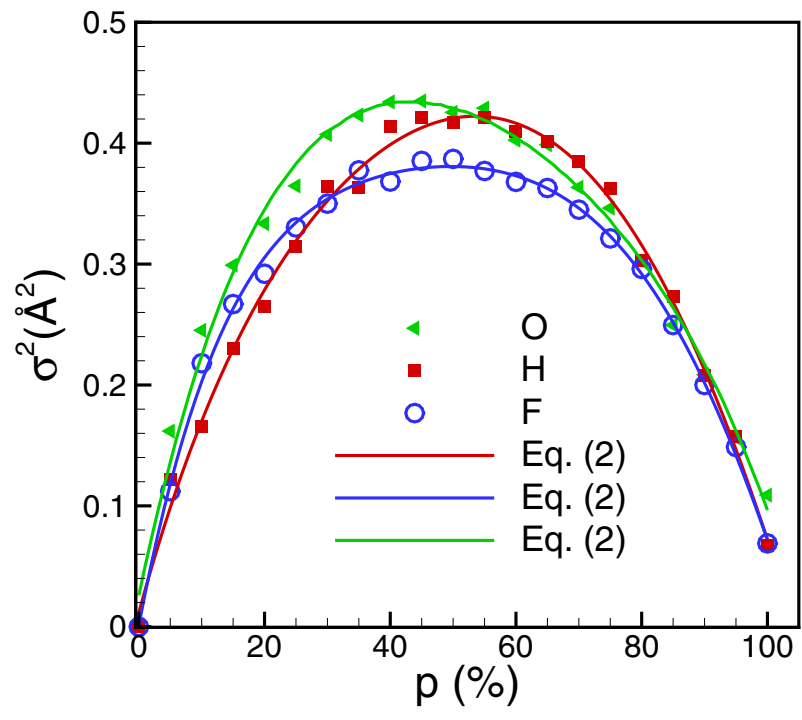

FIG. 3. (Color online) The roughness of partially hydrogenated, fluorinated graphene, and oxidized graphene at $T=0 \mathrm{~K}$.

are able to quantify the rippling of any particular hydrogenated/fluorinated graphene by calculating its roughness. In Fig. 2 we show a typical density plot of the out-of-plane movement of the $\mathrm{C}$ atoms in a system with $50 \%$ hydrogen coverage at zero temperature. The out-of-plane deformations are random and symmetric in the negative and positive $z$ direction, indicating the random out-of-plane movement of the $\mathrm{C}$ atoms. Notice that partially hydrogenated graphene, independent of temperature, is intrinsically a nonflat system. This is due to the fact that the randomly distributed $s p^{3}$ bonds in the system cause local buckling around each C-H/C-F/C-O bond. Note that, in all the simulations, $\langle z\rangle \approx 0$.

The variation of $\sigma^{2}$ with $p$ at $T=0 \mathrm{~K}$ is shown for $\mathrm{H}$, $\mathrm{F}$, and $\mathrm{O}$ functionalized systems in Fig. 3. The maxima are around $p=50 \%$ (symbols). A polynomial fit according to the equation

$$
\sigma^{2}=\sum_{n=0}^{4} a_{n} p^{n}
$$

is shown by the solid curves (where $p$ varies between 0 and 100). The corresponding fitting parameters are listed in Table I for partially hydrogenated (fluorinated and oxidized) graphene. It is interesting to note that these profiles are almost symmetric around $p=50 \%$. The almost quadratic dependence on the roughness influences the other physical properties of partially $\mathrm{H} / \mathrm{F} / \mathrm{O}$ functionalized graphene such as its melting temperature [16]. Assuming that larger roughness results in less stability in the system, we can qualitatively argue that the quadratic dependence of the roughness shows that the system with concentrations close to $0 \%$ and $100 \%$ are more stable. We found indeed that the melting temperature is higher for concentrations that are close to $0 \%$ and $100 \%$.

It is also interesting to investigate the variation of $\sigma^{2}$ with temperature, e.g., for $p=50 \%$, that gives us insight about the thermal rippling effects. We found that, e.g., at room temperature, $\sigma^{2} \simeq 10 \AA^{2}$ and $\simeq 20 \AA^{2}$ for hydrogenated and fluorinated graphene, respectively. These numbers are one order of magnitude larger than that at zero temperature that are shown in Fig. 3. The angstrom size roughness and out-of-plane movements can be measured by atomic force microscopy and scanning tunneling microscopy $[38,39]$. For fully covered systems, we reported previously that at zero temperature the out-of-plane deformation is zero and the systems remain flat up to $1000 \mathrm{~K}[15,32]$.

\section{STABILITY OF PARTIALLY HYDROGENATED AND FLUORINATED GRAPHENE}

Here, we study the variation of the binding energy of the carbon atoms as a function of $p$ for $T=0 \mathrm{~K}$. The binding energy (cohesive energy) is the total potential energy (per atom) of the system at $T=0 \mathrm{~K}$. The total potential energy in ReaxFF comprises ten different terms [27], e.g., bond order and van der Waals (vdW) and Coulomb energy. The binding energy is the difference between the energy of an isolated atom with its energy in the bulk. In Fig. 4(a) we show the average binding energy of a carbon atom $\left(E_{\mathrm{C}}\right)$ in the partially hydrogenated/fluorinated/oxidized systems as a function of $p$. ReaxFF gives $7.87 \mathrm{eV} /$ atom as the binding energy of the $\mathrm{C}$ atom, which is in good agreement with the density functional theory result, i.e., $7.74 \mathrm{eV} /$ atom [40], and is close to the cohesive energy of bulk graphite, i.e., $7.37 \mathrm{eV} /$ atom [40]. We also found the formation energies of fully hydrogenated/fluorinated graphene to be 2.64 and $3.65 \mathrm{eV} /$ atom, respectively, which are in good agreement with the density functional theory (DFT) results, i.e., 2.8 and $3.6 \mathrm{eV} /$ atom [22]. The excellent agreement between DFT and ReaxFF is very promising because ReaxFF allows us to overcome the size limitations of DFT calculations and to perform simulations for thousands of atoms.

The energy of a carbon atom in fluorinated graphene increases slightly with $p$ up to $p=35 \%$, after which it decreases nonlinearly. However, the energy of a carbon atom in the hydrogenated system decreases for all values of $p$. This shows that, independent of $p$, the binding energy of a $\mathrm{C}$ atom in hydrogenated graphene is lower than that of fluorinated graphene (the $\mathrm{C}$-C bonds in hydrogenated graphene are shorter). In oxidized graphene the binding energy increases rapidly, indicating an instability of the armchair structure of oxidized graphene. We conclude that graphene with $p=35 \%$

TABLE I. Fitting parameters of Eq. (2) for different studied systems.

\begin{tabular}{lccrrr}
\hline \hline & $a_{0}$ & $a_{1}$ & \multicolumn{1}{c}{$a_{2}$} & \multicolumn{1}{c}{$a_{3}$} & \multicolumn{1}{c}{$a_{4}$} \\
\hline $\mathrm{O}$ & $2.60 \times 10^{-2}$ & $2.45 \times 10^{-2}$ & $-5.16 \times 10^{-4}$ & $-4.68 \times 10^{-6}$ & $1.90 \times 10^{-8}$ \\
$\mathrm{H}$ & $1.50 \times 10^{-2}$ & $1.76 \times 10^{-2}$ & $-2.53 \times 10^{-4}$ & $1.72 \times 10^{-6}$ & $-8.91 \times 10^{-9}$ \\
$\mathrm{~F}$ & $8.38 \times 10^{-3}$ & $2.39 \times 10^{-2}$ & $-5.7 \times 10^{-4}$ & $6.26 \times 10^{-6}$ & $-2.90 \times 10^{-8}$ \\
\hline \hline
\end{tabular}



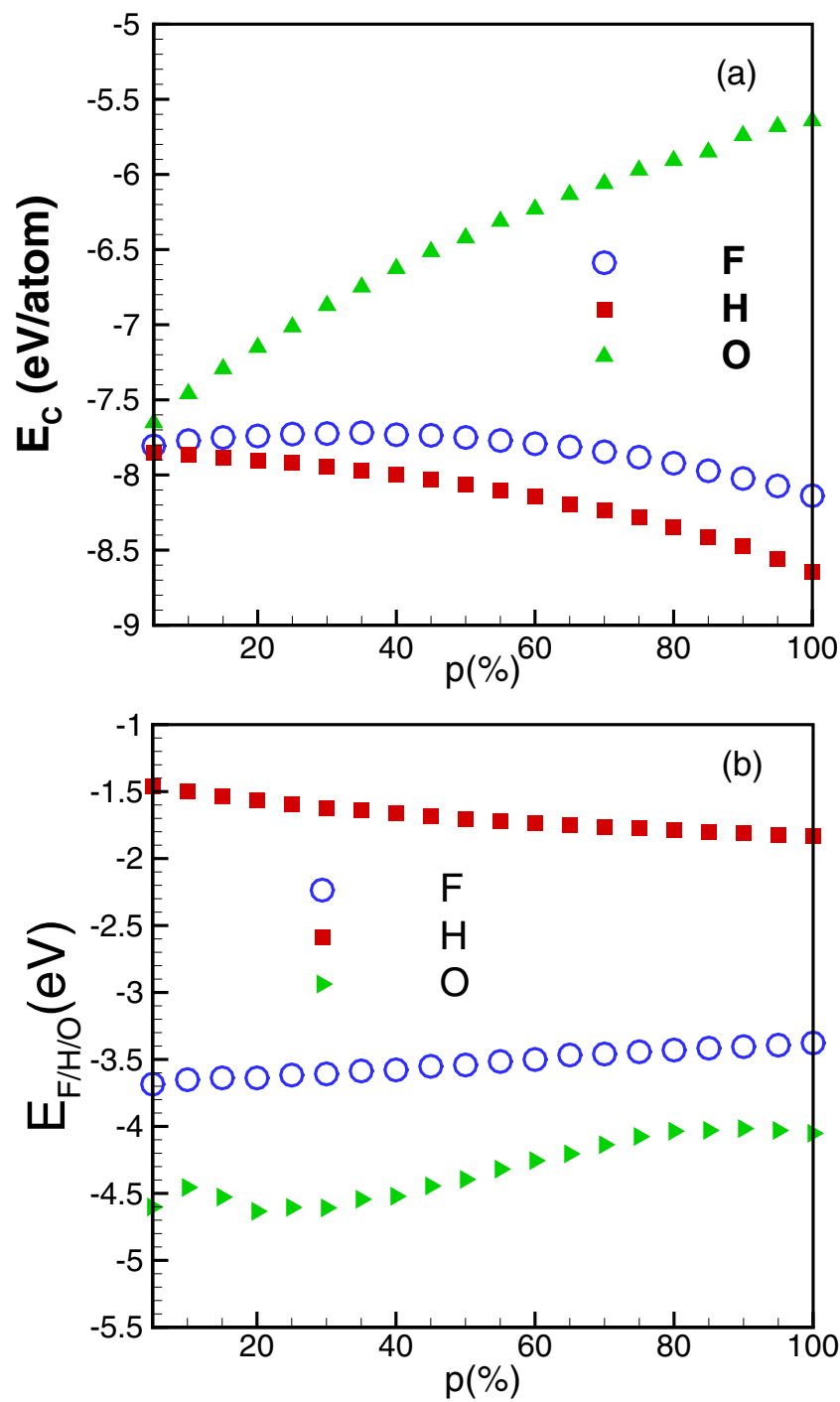

FIG. 4. (Color online) (a) The average binding energy of a $\mathrm{C}$ atom in hydrogenated, fluorinated, and oxidized graphene as a function of the concentration number $p$. (b) The corresponding average energy of $\mathrm{H}, \mathrm{F}$, and $\mathrm{O}$ atoms in, respectively, hydrogenated, fluorinated, and oxidized graphene as a function of the concentration number $p$.

fluorine is less stable than for other $\mathrm{C}: \mathrm{F}$ ratios. In fact, $\mathrm{F}$ atoms absorb more electrons (in contrast to $\mathrm{H}$ ) from the $\mathrm{C}$ atoms and weaken the $\mathrm{C}-\mathrm{C}$ bond. In fully fluorinated (hydrogenated) graphene, the $\mathrm{C}-\mathrm{C}$ and $\mathrm{C}-\mathrm{F}(\mathrm{C}-\mathrm{H})$ bonds are found to be $1.58(1)$ and 1.41(2) $\AA$, respectively [1.56(4) and 1.10(2) $\AA$ ]. These are in agreement with DFT results, i.e., $\mathrm{C}-\mathrm{C}$ and $\mathrm{C}-\mathrm{F}$ are 1.37 and $1.55 \AA$ in fluorinated graphene and $\mathrm{C}-\mathrm{C}$ and $\mathrm{C}-\mathrm{H}$ are 1.52 and $1.12 \AA$ in hydrogenated graphene [22]. The latter confirms the validity of the ReaxFF potential. The different electronegativities of $\mathrm{H}$ and $\mathrm{F}$ atoms are responsible for those changes in the respective chemical bonds. Furthermore, the oxygen atom cannot bind to the carbon atoms in a similar way to which the $\mathrm{H}$ and $\mathrm{F}$ atoms bind.

In Fig. 4(b) we depict the variation of the energy of the H, $\mathrm{F}$, and $\mathrm{O}$ atoms with $p$. Surprisingly, the energy of an $\mathrm{H}$ atom in partially hydrogenated graphene decreases with $p$ while the energy of an $\mathrm{F}$ atom increases almost linearly with $p$ in the whole range of $p$. By increasing $p$, the number of $\mathrm{C}-\mathrm{F}$ bonds increases, which results in weaker $\mathrm{C}-\mathrm{C}$ bonds. These are important results, showing the complexity in the stability of partially hydrogenated and fluorinated graphene. The $\mathrm{O}$ atoms again lose their stability by increasing $p$. The latter confirms the instability of oxidized graphene when it has an armchair structure. Because of this instability, hereafter, we do not continue the study of the armchair structure of oxidized graphene. Indeed, the true structure of oxidized graphene is still a challenge. Different possible structures for graphene oxide can be found in Ref. [41].

\section{SIZE EFFECTS}

We performed additional MD simulations in order to study the size effects of the roughness of partially fluorinated graphene and hydrogenated graphene with $40 \% \mathrm{~F}$ and $\mathrm{H}$, respectively. We generated five samples with sizes $L=$ $\sqrt{L_{\mathrm{zz}} L_{\mathrm{ac}}}=39.7,79.5,119.3,156.8$, and $198.8 \AA$, where $L_{\mathrm{zz}}$ and $L_{\mathrm{ac}}$ are the lengths of the samples in the zigzag and armchair directions, respectively. In Fig. 5 we depict the variation of $\sigma^{2}$ as a function of $L$. It is interesting to note that the roughness of the system is independent of the size. Therefore, at zero temperature there is no size effect in the roughness of the partially hydrogenated and fluorinated graphene. The roughness only depends on the $\mathrm{C}: \mathrm{H}$ or $\mathrm{C}: \mathrm{F}$ ratio while the roughness of graphene and of fully hydrogenated graphene at room temperature depends on the size of the computational unit cell because of the well known anharmonicity in the flexural modes [15].

\section{STATISTICAL ANALYSIS}

Now we show that the random positions for distributed $\mathrm{F}$ and $\mathrm{H}$ over graphene for a given $p$ do not affect the results. The statistical error related to the random distribution of, e.g.,

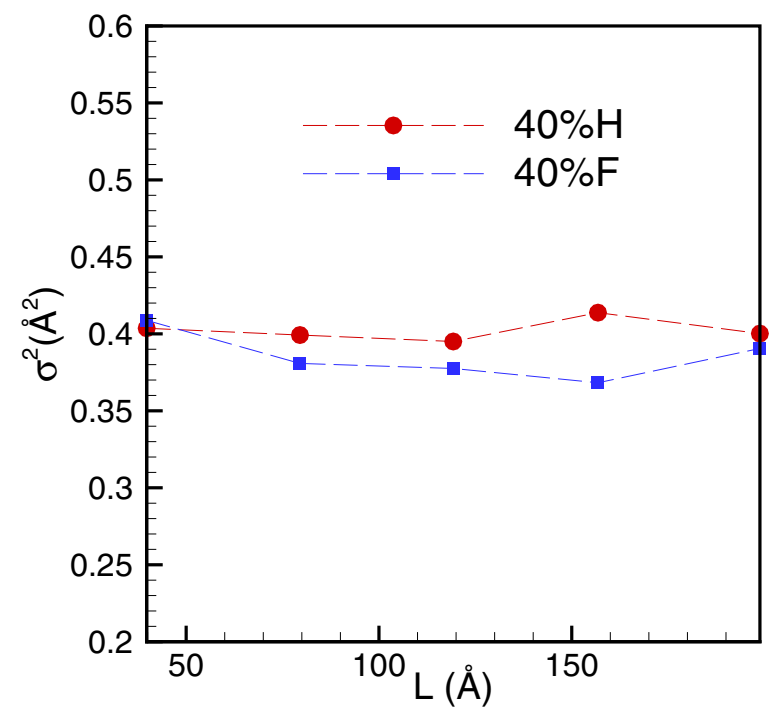

FIG. 5. (Color online) The variation of the roughness of hydrogenated and fluorinated graphene as a function of the size of the unit cell. 


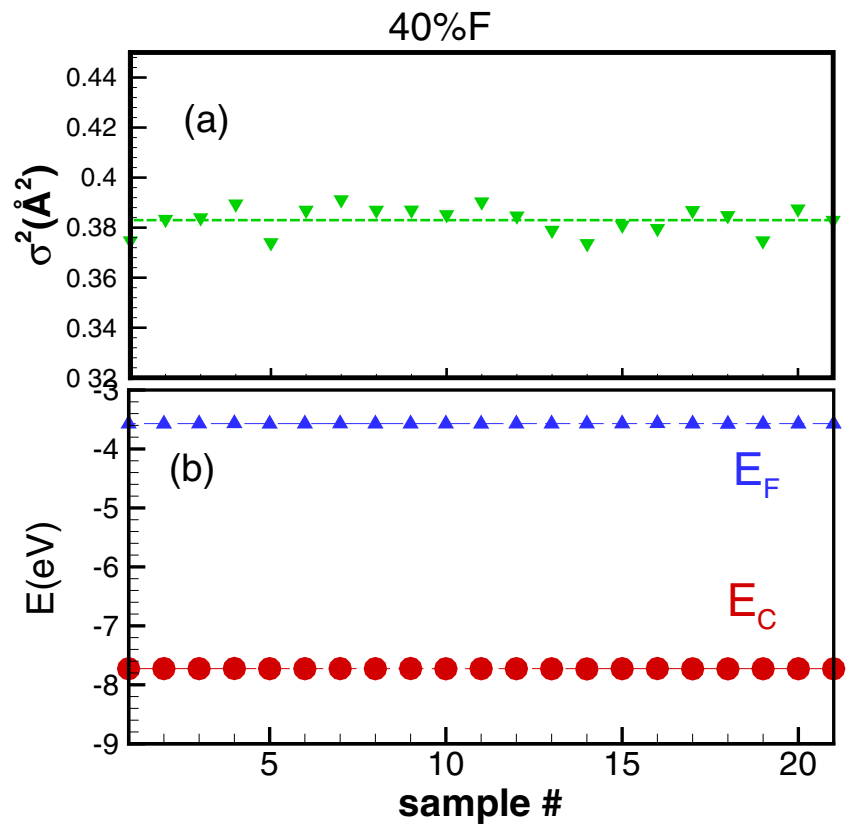

FIG. 6. (Color online) The variation of the roughness, carbon, and fluorine energy in 21 systems, which are all fluorinated graphene $(40 \% \mathrm{~F})$ with different random positions of $\mathrm{F}$ over graphene.

fluorine over graphene for a given $p$ can be obtained by performing several calculations for an ensemble containing 21 systems, all with $40 \%$ fluorination (4500 F atoms and $11200 \mathrm{C}$ atoms) but with random positions of the fluor atoms over graphene. The results for the roughness and the average energy of carbon and fluorine are given in Fig. 6. Notice that the standard deviation in each case is smaller than 0.005, which is clear evidence of the small error of the presented quantities in this study. The roughness was found to be $\sigma^{2}=0.383 \pm 0.005 \AA$, and the average energies of carbon and fluorine were found to be $E_{\mathrm{C}}=-3.572 \pm 0.003 \mathrm{eV} /$ atom and $E_{\mathrm{F}}=-7.726 \pm 0.001 \mathrm{eV} /$ atom, respectively.

\section{LATTICE EXPANSION DUE TO HYDROGENATION/FLUORINATION}

In Figs. 7(a) and 7(b) we depict the variation of the zigzag $\left(L_{\mathrm{zz}}\right)$ and the armchair $\left(L_{\mathrm{ac}}\right)$ size with $p$. In both hydrogenated and fluorinated graphene the zigzag size is almost independent of $p$ in the range $10 \%<p<40 \%$ and it increases linearly beyond it. However, along the armchair direction for fluorinated graphene, a minimum occurs in the size around $40 \%$ while beyond $40 \%$ the armchair size increases with about $8 \AA^{2}$ up to $p=100 \%$. For partially hydrogenated graphene the armchair size is almost constant from $10 \%$ to $40 \%$, and then increases to about $3.5 \AA^{2}$ up to $p=100 \%$. Finally, the projected area of the systems $\left(A_{p}=L_{\mathrm{zz}} L_{\mathrm{ac}}\right)$ is almost constant before $40 \%$ and increases linearly beyond it. These results show that the size of partially functionalized graphene at zero temperature is more or less constant at low concentration (namely, $<40 \%$ ). Hydrogenation increases the size of the system, e.g., for $p=100 \%$ with about $8 \%$ and for the fluorinated system it increases the size of the system,
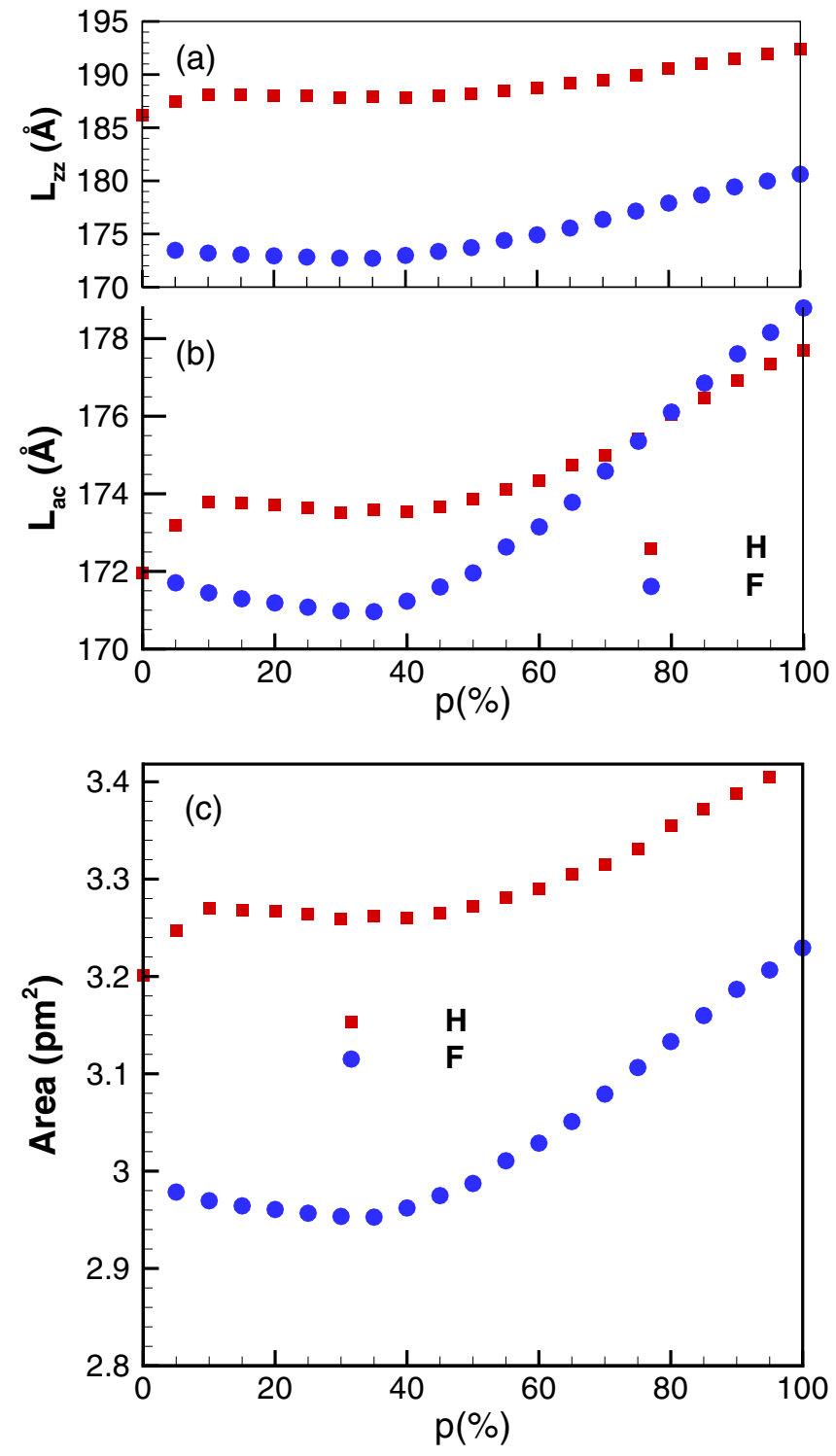

FIG. 7. (Color online) (a) The zigzag and (b) armchair size variations with $p$ in $\mathrm{H}$ and $\mathrm{F}$ functionalized graphene. (c) The variations of the area of the unit cell of $\mathrm{H}$ and $\mathrm{F}$ functionalized graphene with $p$.

e.g., at $p=100 \%$ with about $10 \%$. The latter is due to the weakening of the $\mathrm{C}-\mathrm{C}$ bonds in the presence of $\mathrm{H}$ or $\mathrm{F}$.

\section{LATTICE THERMAL CONTRACTION}

Employing the heating process in MD simulations, we study the lattice deformation of partially functionalized graphene where we take the concentration $p=40 \%$ as an example. In Fig. 8 we show the length and width of the system corresponding to the armchair and zigzag directions in the systems as a function of temperature. Both $L_{\mathrm{zz}}$ and $L_{\mathrm{ac}}$ decrease with temperature up to $200 \mathrm{~K}$ and then oscillate beyond it. We also calculated the corresponding binding energies with temperature (see Fig. 9). The variation of the binding energy of the carbon increases linearly, consistent with the well known behavior of classical systems (e.g., the energy 


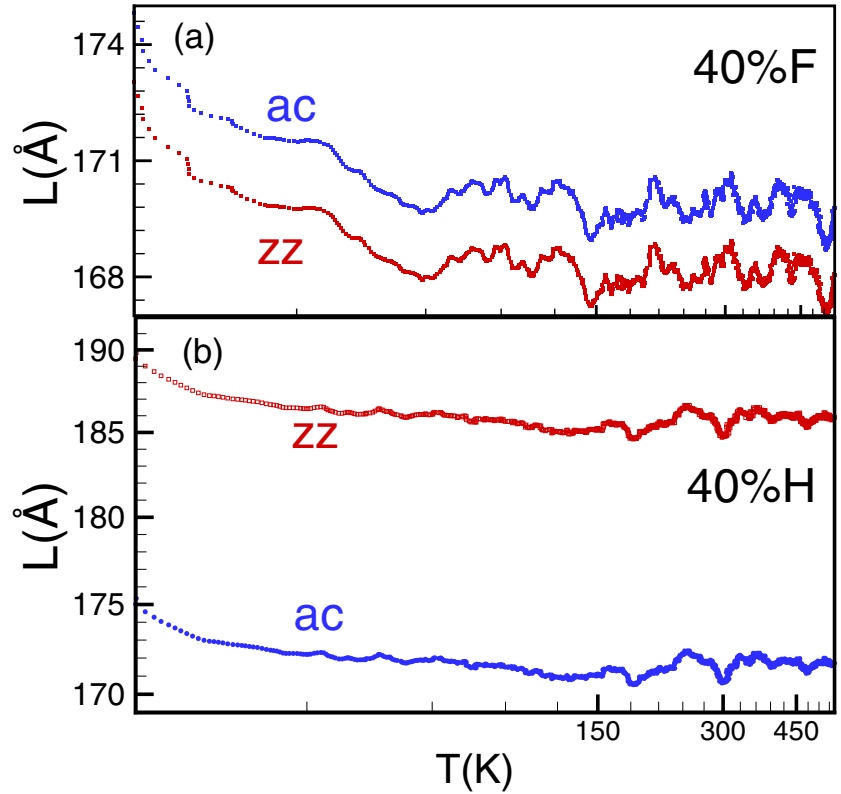

FIG. 8. (Color online) The zigzag and armchair lengths of functionalized graphene with $40 \%$ fluorine (a) and hydrogen (b) as functions of temperature.

of an ideal classical gas). There is a clear difference in the binding energy of $\mathrm{H}$ and $\mathrm{F}$ atoms. The binding energy of the $\mathrm{F}$ atom remains constant below $200 \mathrm{~K}$, but it increases beyond $200 \mathrm{~K}$. This can be due to the strong C-F bond in comparison to the $\mathrm{C}-\mathrm{H}$ bond.

Finally, we can estimate the lattice thermal expansion by calculating the change in the projected area of the computational unit cell $\left[A_{p}(T)=L_{\mathrm{zz}} L_{\mathrm{ac}}\right]$ with temperature

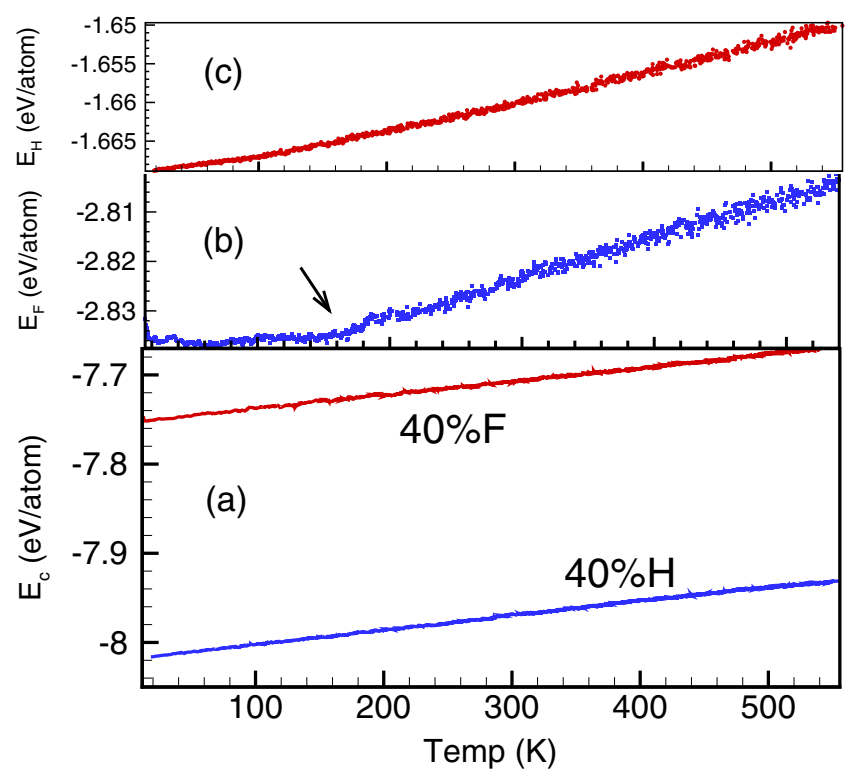

FIG. 9. (Color online) The variation of the binding energy of (a) carbon, (b) fluorine, and (c) hydrogen with temperature in hydrogenated and fluorinated graphene with $40 \%$ hydrogen and fluorine.

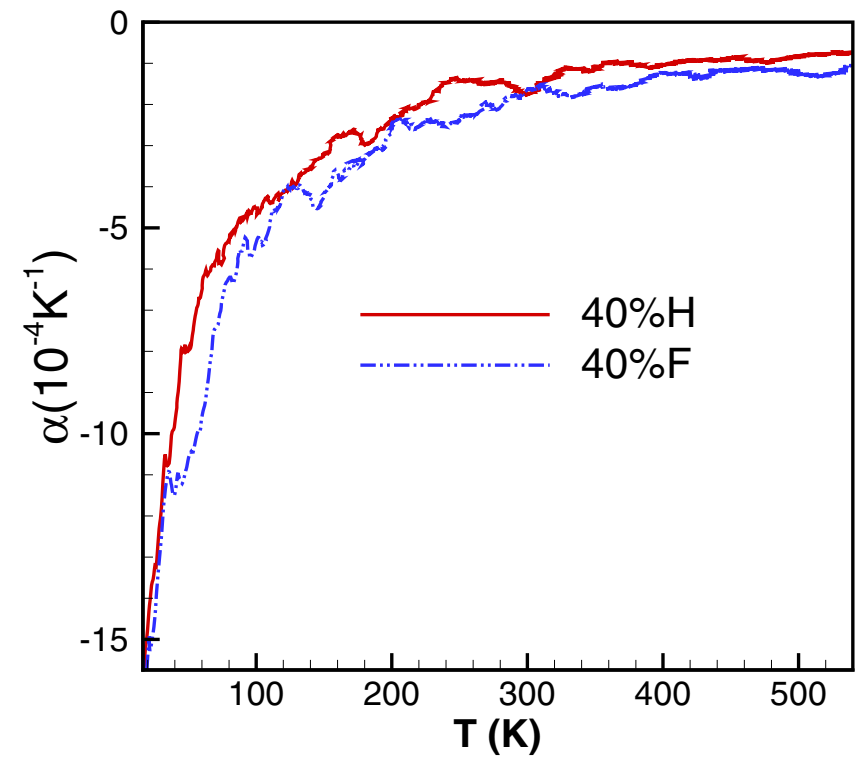

FIG. 10. (Color online) The variation of the linear thermal expansion of functionalized graphene with $40 \%$ hydrogen and fluorine. The error in the estimation of $\alpha$ is less than $0.1 \%$.

by using

$$
\alpha=\frac{d A_{p}}{A_{p}(0) d T} .
$$

We found a $2.65 \%$ and $2.64 \%$ (2.84\% and $3.16 \%$ ) reduction in the armchair and zigzag size in fluorinated (hydrogenated) graphene with $p=40 \%$, respectively. Using Eq. (3), the results for the projected area as functions of temperature are shown in Fig. 10. It is interesting to note that $\alpha$ for fluorinated graphene is slightly lower than for hydrogenated graphene in the whole temperature range. Moreover, the results at room temperature are one order of magnitude larger than those for fully hydrogenated graphene [42] and for pristine graphene, i.e., $-(4 \pm 1) \times 10^{-6} \mathrm{~K}^{-1}$. Therefore, it should be easier to measure the thermal contraction in partially hydrogenated or fluorinated graphene.

\section{DISCUSSIONS AND CONCLUSIONS}

Using state-of-the art MD simulations we performed extensive simulations to study the structural and thermal properties of partially and fully hydrogenated and fluorinated graphene. There are two basic mechanisms to tune the electronic gap in graphene: (i) disturbing the band crossing at the Dirac points by breaking the equivalence of two sublattices, or (ii) transforming the carbon hybridization from $s p^{2}$ into $s p^{3}$ via chemical functionalization. For instance, using Raman spectroscopy, Withers et al. [43] found that, depending on the fluorine concentration, different transport regimes can be accessed. In an experimental report on hydrogenated [13] and fluorinated [18] graphene, partially fluorinated graphene was found to be unstable if graphene was fluorinated only for a few hours. The latter process was found to be largely reversible, but after more extensive fluorination (about 1 day), the process was found to be irreversible. These effects can be elucidated by the structural stability of the partially functionalized graphene. 


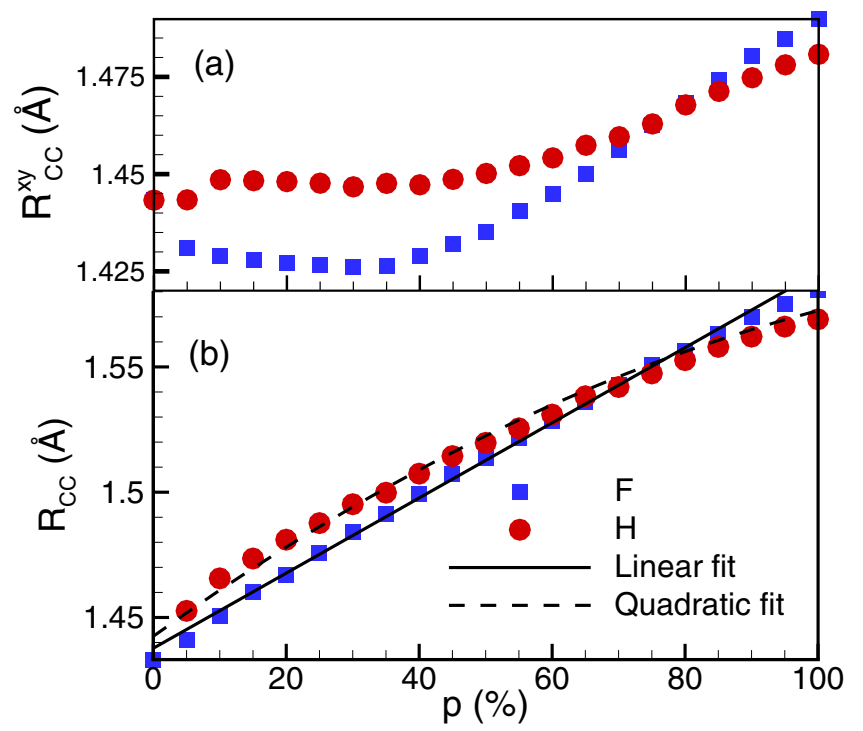

FIG. 11. (Color online) (a) The variation of the (a) in-plane C-C distance and (b) $\mathrm{C}-\mathrm{C}$ bond length with $\mathrm{C}: \mathrm{H}$ and $\mathrm{C}: \mathrm{F}$ ratio percentages in functionalized graphene by hydrogen and fluorine.

Here, we showed that by increasing the $\mathrm{C}: \mathrm{H}$ or $\mathrm{C}: \mathrm{F}$ ratios, the binding energy of carbon decreases, which results in a larger formation energy and in a mechanically more stable system with larger $\mathrm{C}: \mathrm{H}$ and $\mathrm{C}: \mathrm{F}$ ratios [see Fig. 4(a)]. This may explain the aforementioned experiment. Even at $0 \mathrm{~K}$, starting from zero concentration of $\mathrm{H}$ and $\mathrm{F}$ up to $p=40 \%-50 \%$ concentration, the systems deviate from a flat structure, but it becomes almost flat when $p$ approaches $p=100 \%$. In fact, the system transits from an ordered phase to maximal disordered phase when $p=40 \%-50 \%$ and returns to a new ordered phase at $p=100 \%$. The size of the roughness of the system can be understood as an order parameter.

The sizes of the samples along the zigzag or armchair directions change differently depending on the type of added atom. This is because of the different electronic structure of the $\mathrm{F}$ and $\mathrm{H}$ atom, where one absorbs electrons and the other loses electrons. The resulted buckling structures due to the presence of $\mathrm{H} / \mathrm{F}$ change the local areas along the zigzag and armchair directions differently. This nonuniform out-of-plane buckling in the partially functionalized graphene is the origin of the observed effects, i.e., the $\mathrm{C}-\mathrm{C}$ bond in the partially functionalized system is varying over the system. The net area of the sample beyond $p=40 \%$ increases with $p$ in both fluorinated and hydrogenated graphene, which is due to the elongation of the $\mathrm{C}-\mathrm{C}$ bonds. We show the variation of the averaged in-plane distance $\left(R_{\mathrm{CC}}^{x y}\right)$ between the $\mathrm{C}$ atoms in Fig. 11(a) and the averaged $\mathrm{C}-\mathrm{C}$ bond lengths in Fig. 11(b) for hydrogenated (red dots) and fluorinated (square symbols) graphene as a function of $p$ (for any given $p$ the averaging is done over the minimum energy configurations). The solid line in Fig. $11(\mathrm{~b})$ is a linear fit $\left(R_{\mathrm{CC}}=1.5 \times 10^{-3} p+1.438\right)$ of the $\mathrm{C}-\mathrm{C}$ bond length data for fluorinated graphene and the dashed line is a quadratic fit $\left(R_{\mathrm{CC}}=-6.0 \times 10^{-6} p^{2}+\right.$ $1.9 \times 10^{-3} p+1.443$ ) of the $\mathrm{C}-\mathrm{C}$ bond length data for the hydrogenated graphene. The variation of in-plane $\mathrm{C}-\mathrm{C}$ distance with $p$ [Fig. 11(a)] explains the results for the in-plane lengths, i.e., $L_{\mathrm{zz}}$ and $L_{\mathrm{ac}}$, as functions of $p$ [see Figs. 8(a) and 8(b)]. Notice that although $R_{\mathrm{CC}}$ increases with $p$, it is not directed in plane, especially when $p$ is larger than $40 \%$.

We also found that by heating the system up to $550 \mathrm{~K}$, the binding energy of $\mathrm{C}$ atoms increases linearly with temperature, which is in agreement with a typical classical system [42]. The projected area of the sample decreases with temperature up to $200 \mathrm{~K}$, and after this temperature it starts to oscillate around the new length. This shows the negative thermal expansion in partially functionalized graphene.

\section{ACKNOWLEDGMENT}

This work was supported by the Flemish Science Foundation (FWO-Vl) and the Methusalem Foundation of the Flemish Government.
[1] K. S. Novoselov, A. K. Geim, S. V. Morozov, D. Jiang, Y. Zhang, S. V. Dubonos, I. V. Grigorieva, and A. A. Firsov, Science 306, 666 (2004).

[2] A. K. Geim and K. S. Novoselov, Nat. Mater. 6, 186 (2007).

[3] A. K. Geim, Science 324, 1530 (2009).

[4] C. Xu, H. Li, and K. Banerjee, IEEE Trans. Electron Devices 55, 3246 (2008).

[5] F. Schedin, A. K. Geim, S. V. Morozov, E. W. Hill, P. Blake, M. I. Katsnelson, and K. S. Novoselov, Nat. Mater. 6, 652 (2007).

[6] C. Gomez-Navarro, R. T. Weitz, A. M. Bittner, M. Scolari, A. Mews, M. Burghard, and K. Kern, Nano Lett. 7, 3499 (2007).

[7] T. J. Booth, P. Blake, R. R. Nair, D. Jiang, E. W. Hill, U. Bangert, A. Bleloch, M. Gass, K. S. Novoselov, M. I. Katsnelson, and A. K. Geim, Nano Lett. 8, 2442 (2008).

[8] F. Wang, Y. B. Zhang, C. S. Tian, C. Girit, A. Zettl, M. Crommie, and Y. R. Shen, Science 320, 206 (2008).

[9] A. A. Balandin, S. Ghosh, W. Z. Bao, I. Calizo, D. Teweldebrhan, F. Miao, and C. N. Lau, Nano Lett. 8, 902 (2008).
[10] J. C. Meyer, A. Geim, M. I. Katsnelson, K. S. Novoselov, T. J. Booth, and S. Roth, Nature (London) 446, 60 (2007); D. A. Kirilenko, A. T. Dideykin, and G. Van Tendeloo, Phys. Rev. B 84, 235417 (2011).

[11] S. K. Singh, M. Neek-Amal, S. Costamagna, and F. M. Peeters, Phys. Rev. B 87, 184106 (2013).

[12] G. A. Olah and A. Molnar, Hydrocarbon Chemistry (Wiley Interscience, Hoboken, NJ, 2003).

[13] D. C. Elias, R. R. Nair, T. M. G. Mohiuddin, S. V. Morozov, P. Blake, M. P. Halsall, A. C. Ferrari, D. W. Boukhvalov, M. I. Katsnelson, A. K. Geim, and K. S. Novoselov, Science 323, 610 (2009).

[14] M. Z. S. Flores, P. A. S. Autreto, S. B. Legoas, and D. S. Galvao, Nanotechnology 20, 465704 (2009).

[15] S. Costamagna, M. Neek-Amal, J. H. Los, and F. M. Peeters, Phys. Rev. B 86, 041408(R) (2012).

[16] S. K Singh, S. Costamagna, M. Neek-Amal, and F. M. Peeters, J. Phys. Chem. C 118, 4460 (2014).

[17] H. Sahin, O. Leenaerts, S. K. Singh, and F. M. Peeters, Wiley Interdiscip. Rev.: Comput. Mol. Sci. 5, 255 (2015). 
[18] R. Nair, W. Ren, R. Jalil, I. Riaz, V. Kravets, L. Britnell, P. Blake, F. Schedin, A. Mayorov, S. Yuan, M. Katsnelson, H. Cheng, W. Strupinski, L. Bulusheva, A. Okotrub, I. Grigorieva, A. Grigorenko, K. Novoselov, and A. K. Geim, Small 6, 2877 (2010).

[19] J. O. Sofo, A. S. Chaudhari, and G. D. Barber, Phys. Rev. B 75, 153401 (2007).

[20] O. Leenaerts, H. Peelaers, A. D. Hernández-Nieves, B. Partoens, and F. M. Peeters, Phys. Rev. B 82, 195436 (2010).

[21] D. W. Boukhvalov, M. I. Katsnelson, and A. I. Lichtenstein, Phys. Rev. B 77, 035427 (2008).

[22] H. Sahin, M. Topsakal, and S. Ciraci, Phys. Rev. B 83, 115432 (2011).

[23] R. J. Kashtiban, M. A. Dyson, R. R. Nair, R. Zan, S. L. Wong, Q. Ramasse, A. K. Geim, U. Bangert, and J. Sloan, Nat. Commun. 5, 4902 (2014).

[24] M. Neek-Amal, J. Beheshtian, F. Shayeganfar, S. K. Singh, J. H. Los, and F. M. Peeters, Phys. Rev. B 87, 075448 (2013).

[25] W. H. Lee, J. W. Suk, H. Chou, J. Lee, Y. Hao, Y. Wu, R. Piner, D. Akinwande, K. S. Kim, and R. S. Ruoff, Nano Lett. 12, 2374 (2012).

[26] J. T. Robinson, J. S. Burgess, C. E. Junkermeier, S. C. Badescu, T. L. Reinecke, F. K. Perkins, M. K. Zalalutdniov, J. W. Baldwin, J. C. Culbertson, and P. E. Sheehan, Nano Lett. 10, 3001 (2010).

[27] A. C. T. van Duin, S. Dasgupta, F. Lorant, and W. A. Goddard, J. Phys. Chem. A 105, 9396 (2001).

[28] A. C. T. van Duin and J. S. S. Damsté, Org. Geochem. 34, 515 (2003).

[29] K. Chenoweth, A. C. T. van Duin, and W. A. Goddard, III, J. Phys. Chem. A 112, 1040 (2008).
[30] S. Plimpton, J. Comput. Phys. 117, 1 (1995).

[31] See http://lammps.Sandia.gov for more information about LAMMPS.

[32] S. K. Singh, S. G. Srinivasan, M. Neek-Amal, S. Costamagna, A. C. T. van Duin, and F. M. Peeters, Phys. Rev. B 87, 104114 (2013).

[33] K. Chenoweth, S. Cheung, A. C. T. van Duin, W. A. Goddard, and E. M. Kober, J. Am. Chem. Soc. 127, 7192 (2005).

[34] N. V. Medhekar, A. Ramasubramaniam, R. S. Ruoff, and V. B. Shenoy, ACS Nano 4, 2300 (2010).

[35] A. Bagri, C. Mattevi, M. Acik, Y. J. Chabal, M. Chhowalla, and V. B. Shenoy, Nat. Chem. 2, 581 (2010).

[36] S. Nosé, J. Chem. Phys. 81, 511 (1984).

[37] W. G. Hoover, Phys. Rev. A 31, 1695 (1985).

[38] W. Yang, G. Chen, Z. Shi, C.-C. Liu, L. Zhang, G. Xie, M. Cheng, D. Wang, R. Yang, D. Shi, K. Watanabe, T. Taniguchi, Y. Yao, Y. Zhang, and G. Zhang, Nat. Mater. 12, 792 (2013).

[39] L. Zhao, R. He, K. T. Rim, T. Schiros. K. S. Kim, H. Zhou, C. Gutierrez, S. P. Chockalingam, C. J. Arguello, L. Palova, D. Nordlund, M. S. Hybertsen, D. R. Reichman, T. F. Heinz, P. Kim, A. Pinczuk, G. W. Flynn, and A. N. Pasupathy, Science 333, 999 (2011).

[40] H. Shin, S. Kang, J. Koo, H. Lee, J. Kim, and Y. Kwon, J. Chem. Phys. 140, 114702 (2014).

[41] D. R. Dreyer, S. Park, C. W. Bielawski, and R. S. Ruof, Chem. Soc. Rev. 39, 228 (2010).

[42] M. Neek-Amal and F. M. Peeters, Phys. Rev. B 83, 235437 (2011).

[43] F. Withers, S. Russo, M. Dubois, and M. F. Craciun, Nanoscale Res. Lett. 6, 526 (2011). 